\title{
Study of Relationship between Central Venous Pressure and Peripheral Venous Pressure during Intraoperative Period in Neurosurgical Patients
}

\author{
Jay Prakash ${ }^{1} \quad$ Natesh S. Rao ${ }^{1}$ Sanjeev Kumar ${ }^{2} \quad$ Kumar H. Raghwendra ${ }^{2} \quad K^{\prime}$ hushboo Saran ${ }^{3}$ Sandeep D. Reddy ${ }^{1}$
}

\author{
${ }^{1}$ Department of Anaesthesia and Critical Care Medicine, Vydehi \\ Institute of Medical Sciences and Research Centre, Bengaluru, India \\ ${ }^{2}$ Department of Anaesthesia and Critical Care Medicine, Indira \\ Gandhi Institute of Medical Sciences, Patna, Bihar, India \\ ${ }^{3}$ Department of Pathology, Vydehi Institute of Medical Sciences and \\ Research Centre, Bengaluru, India
}

J Neuroanaesthesiol Crit Care 2018;5:15-20.

\begin{abstract}
Address for correspondence Jay Prakash, MD, Department of Anaesthesia and Critical Care Medicine, Vydehi Institute of Medical Sciences and Research Centre, 205, Roma Pearl Apartment, AECS Layout A Block, Kundalahalli, Bengaluru-560037, India (e-mail: dr.jay_prakash@rediffmail.com).
\end{abstract}

\begin{abstract}
Keywords

- central venous pressure

- peripheral venous pressure

- neurosurgery

- hemodynamics

- monitoring

Background and Objectives Central venous pressure (CVP) and peripheral venous pressure (PVP) are strongly correlated during various surgeries. This study was designed to examine the consistency of CVP-PVP relationships in circumstances of rapidly fluctuating hemodynamics in neurosurgical patients. Prime objective of this study was to determine if PVP can be an effective alternative to invasive CVP for assessing volume status during neurosurgical procedures when expertise, equipment, and patient's condition contraindicate invasive monitoring.

Subjects and Methods After the approval by the Institutional Ethics Committee, CVP and PVP were measured in 50 neurosurgical patients of the American Society of Anesthesiologists grade I and II operated in supine position. Paired measurements of CVP and PVP were made every 20 minutes, from the starting of anesthesia until the end of surgery; however, in situations of hemodynamic instability, the readings were taken every 5 minutes of interval.

Results The study showed a strong correlation between CVP and PVP (Pearson's correlation coefficient between CVP and PVP, $r=0.89 ; 95 \% \mathrm{Cl}: 0.81-0.93 ; p<0.001$ ). Mean CVP was $5.7 \pm 0.8 \mathrm{~mm}$ of $\mathrm{Hg}$, mean PVP was $10.4 \pm 0.6 \mathrm{~mm}$ of $\mathrm{Hg}$, and bias between CVP and PVP was $4.7 \pm 0.4$ ( $95 \% \mathrm{Cl}:-4.61$ to -4.83 ). The Bland-Altman analysis showed that limit of agreement to be 4.0 to $5.5 \mathrm{~mm}$ of $\mathrm{Hg}$.

Conclusion This study demonstrated a strong correlation between CVP and PVP. Therefore, PVP monitoring may be a reliable alternative to CVP monitoring during neurosurgery.
\end{abstract}

\section{Introduction}

Patients undergoing neurosurgery are at risk of significant blood loss and resulting hemodynamic changes. In case of sudden blood loss, volume of blood in arteries is maintained at expense of that in veins, manifesting as low central venous pressure (CVP) ${ }^{1}$ associated with tachycardia initially and, a fall in arterial pressure is a late sign of hypovolemia. Monitoring of CVP is, therefore, of great assistance during hypovolemia.
CVP is the pressure within the intrathoracic venae cavae, measured by insertion of catheter via the internal jugular or subclavian vein, which is normally equal to the right atrial pressure, unless there is obstruction in the venae cavae. The value used in clinical practice is the pressure recorded at the base of c-wave, at the end of expiration, while the subject is supine. This represents the pressure in the right atrium immediately before the start of ventricular systole. ${ }^{2}$ CVP is often used to estimate right ventricular preload, which
DOI https://doi.org/ $10.1055 / \mathrm{s}-0037-1618328$ ISSN 2348-0548.
Copyright @2018 Indian Society of Neuroanaesthesiology and Critical Care
License terms

(®) (1) $\Theta \circledast$ 
serves as a surrogate for intravascular volume and can help to guide fluid management. ${ }^{3}$ Normal values vary with the context of measurement because right atrial pressure is not constant but varies with the cardiac cycle, with respiration and on change of position between upright and recumbent. However, complications associated with central venous cannulation, such as accidental arterial puncture, arrhythmias, hematoma, pneumothorax, nerve injury, arteriovenous fistula, air embolism, catheter or wire shearing, and infection, can outweigh its benefits. ${ }^{4}$ On the other hand, peripheral venous pressure (PVP) is devoid of such complications and measures the diastolic filling pressure of right heart, that is, pressure in the right atrium. Maintenance of the venous return entails a constantly decreasing pressure in the systemic veins from the periphery toward the heart, so a gradient exists between the pressure measured in any peripheral vein and the pressure which is to be ascertained. ${ }^{5}$

A sudden massive blood loss, resulting in hemodynamic fluctuations requires rapid transfusion guided by CVP monitoring. Besides, neurosurgical patients may also develop hypovolemia from administration of hyperosmolar crystalloids for brain relaxation. ${ }^{6,7}$ Efficient management of hypovolemia from these causes is also aided by CVP monitoring. Literature search revealed only two studies in neurosurgical patients operated for craniotomy, ${ }^{8,9}$ one of which is very small in which the authors compared CVP and PVP in eight patients only and showed good correlation between the two measurements.

We, therefore, conducted this study in neurosurgical patients undergoing craniotomy to confirm if such relationship exists between CVP and PVP values during periods of normal as well as disturbed hemodynamics.

\section{Subjects and Methods}

Approval from the Institutional Ethics Committee was obtained vide letter no. 1052/2014 dated September 26, 2014 and the study was registered with Clinical Trials RegistryIndia (CTRI/2015/09/006230). The study conformed to the Helsinki declaration (World Medical Association, 1995). Written informed consent from each patient or next of kin was taken before enrolment in the study.

This observational study was conducted on 50 patients of the American Society of Anesthesiologists grade I or II, age 21 to 60 years and included both genders. The exclusion criteria were patients with cardiorespiratory comorbidity, coagulation disorder, morbid obesity (body mass index $>30$ ), anticipated difficult peripheral and central venous access, infection at the site of insertion, and thyromegaly or prior neck surgery. Following placement of standard monitors electrocardiogram (ECG), pulse oximeter and noninvasive blood pressure (NIBP) anesthesia was induced with fentanyl and propofol, and tracheal intubation facilitated with rocuronium. Following intubation, a triple lumen central venous catheter was inserted in right jugular vein and sutured to the skin between 16 and $18 \mathrm{~cm}$ mark, which was confirmed postoperatively by chest X-ray. To monitor PVP, an 18G cannula was inserted in the right cephalic vein.
Additional monitoring after tracheal intubation consisted of end tidal $\mathrm{CO}_{2}$, invasive arterial pressure, oesophageal temperature, and urine output. The CVP and PVP were monitored simultaneously in all patients throughout the surgical period including, periods of significant hemodynamic changes. Both CVP and PVP transducers were set to zero at midaxillary line in the fourth intercostal space. Mechanical ventilation was instituted without positive end expiratory pressure (PEEP). Anesthesia was maintained with oxygen, nitrous oxide, isoflurane, fentanyl and rocuronium. On completion of surgery, trachea was extubated after reversal of residual effect of rocuronium.

The measurement arm was abducted 90 degrees on an arm board, protected against external pressure, and kept visible during surgery. Drugs and fluids were not administered through the PVP cannula, and NIBP was measured on the other arm to avoid hindrance to venous flow. Real-time waveforms and numeric pressure values were displayed throughout the case on monitor. Paired simultaneous measurements of CVP and PVP in mm of Hg were made every 20 minutes after tracheal intubation and every 5 minutes whenever there was severe blood loss resulting in hemodynamic instability, until the end of surgery.

Statistical Method: The consistency of CVP-PVP difference between subjects was analyzed by testing Pearson's correlation coefficient. The statistical method for comparison of CVP and PVP was based on the Bland-Altman technique. Limits of agreement, which are defined as \pm 1.96 standard deviation (SD) from the mean difference (d), were studied by Bland Altman plots, and if the difference was normally distributed, the $95 \%$ limit of agreement was $d \pm 1.96$ SD. The precision of the estimated limit of agreement was provided by a $95 \%$ confidence interval (CI). Bias was calculated as the mean of the difference between the simultaneous CVP and PVP measurements. Regression analysis was used to establish the correlation between CVP and PVP. Statistical analysis was performed using the GraphPad InStat version 3.10 (created July 10, 2009) by data entered in the Microsoft Excel spread sheet. A p-value less than 0.05 was considered significant.

\section{Results}

Fifty patients, 36 males (72\%) and 14 females (28\%), were included in the study. Their mean age and body weight were $40.2 \pm 11.0$ years and $55.7 \pm 7.0 \mathrm{~kg}$, respectively. (-Table $\mathbf{1}$ ) summarizes the demographic and clinical characteristics of patients, and ( - Table 2) displays the types of lesions. A total of 626 paired sets of CVP and PVP were taken simultaneously in 50 patients, and 120 simultaneous observations were recorded during hemodynamic instability. Overall, the mean CVP was $5.7 \pm 0.79 \times 1.96 \mathrm{~mm}$ of $\mathrm{Hg}$ and mean PVP was $10.43 \pm 0.57 \times 1.96 \mathrm{~mm}$ of $\mathrm{Hg}$. Bias between CVP and PVP was $4.72 \pm 0.4$ (95\% CI: - 4.61- -4.83). (-Table 3) shows the trend of CVP, PVP, mean arterial pressure, and heart rate. ( - Fig. 1) shows the paired measurement of mean CVP and mean PVP for each of 50 patients. The Bland-Altman analysis showed that limit of agreement between CVP and PVP to be from 3.96 to $5.48 \mathrm{~mm}$ of $\mathrm{Hg}$, and Pearson's correlation coefficient $(r)$ between CVP and PVP was 0.89 (95\% CI: 0.81-0.93, 
Table 1 Demographic and clinical characteristics of patients

\begin{tabular}{|l|l|l|}
\hline & Mean \pm SD & Range \\
\hline Age $(\mathrm{y})$ & $40.22 \pm 11.09$ & $21-60$ \\
\hline Body weight $(\mathrm{kg})$ & $55.74 \pm 6.94$ & $41-70$ \\
\hline ASA (I/II) & $31 / 19$ & \\
\hline Gender (male/female) & $36 / 14$ & \\
\hline Crystalloid (mL) & $1,808 \pm 475.7$ & $1,200-3,000$ \\
\hline Colloid (mL) & $562.5 \pm 165.4$ & $500-1,000$ \\
\hline Estimated blood loss (mL) & $328.6 \pm 254.6$ & $100-1,200$ \\
\hline
\end{tabular}

Abbreviations: ASA, American Society of Anesthesiologists; SD, standard deviation.

Table 2 Types of lesions

\begin{tabular}{|l|l|}
\hline Contusion & 15 \\
\hline Extradural hematoma & 13 \\
\hline Subdural hematoma & 10 \\
\hline Space occupying lesion & 8 \\
\hline Cerebellopontine angle tumor & 2 \\
\hline Frontal space occupying lesion & 2 \\
\hline
\end{tabular}

Table 3 Trends of CVP, PVP, and MAP in $\mathrm{mm}$ of $\mathrm{Hg}$ and HR

\begin{tabular}{|l|l|l|l|l|}
\hline & CVP & PVP & MAP & HR \\
\hline 1st, 20 min & $5.38 \pm 0.75$ & $10.24 \pm 0.72$ & $93 \pm 10$ & $78.04 \pm 14.46$ \\
\hline 2nd, 40 min & $5.64 \pm 0.94$ & $10.22 \pm 0.91$ & $90 \pm 9$ & $79.48 \pm 15.59$ \\
\hline 3rd, 1h & $5.76 \pm 0.85$ & $10.54 \pm 0.79$ & $88.26 \pm 9$ & $80.08 \pm 16.81$ \\
\hline 4th, 1.20 h & $5.96 \pm 0.86$ & $10.64 \pm 0.80$ & $88.18 \pm 9.5$ & $82.04 \pm 18.75$ \\
\hline 5th, 1.40 h & $5.83 \pm 0.90$ & $10.54 \pm 0.96$ & $87.93 \pm 8.9$ & $81.56 \pm 18.86$ \\
\hline 6th, 2 h & $5.79 \pm 0.73$ & $10.62 \pm 0.70$ & $89 \pm 9.6$ & $90.54 \pm 17.11$ \\
\hline 7th, 2.20 h & $5.87 \pm 0.81$ & $10.69 \pm 0.70$ & $86.2 \pm 8.2$ & $94.5 \pm 16.04$ \\
\hline 8th, 2.40 h & $6.11 \pm 0.78$ & $11 \pm 0.87$ & $83.7 \pm 7.34$ & $99.11 \pm 15.03$ \\
\hline 9th, 3 h & $5.86 \pm 0.39$ & $10.71 \pm 0.49$ & $86 \pm 8$ & $97.14 \pm 15.87$ \\
\hline 10th, 3.20 h & 5 & 10 & 101 & 108 \\
\hline
\end{tabular}

Abbreviations: CVP, central venous pressure; HR, heart rate; MAP, mean arterial pressure; PVP, peripheral venous pressure.

$p<0.001)$. The correlation improved in case of blood loss more than $600 \mathrm{~mL}$ (estimated blood loss $>600 \mathrm{~mL} ; r=0.87$, 95\% CI: $0.52-0.97$ and $p=0.001$ ). ( - Table 4) shows the paired simultaneous reading of mean CVP and PVP during hemody-

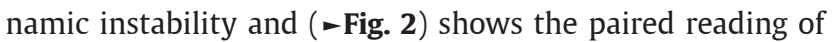
mean CVP and mean PVP during hemodynamic instability. A scatter plot of CVP and PVP with a regression line and line of agreement showed a strong linear relationship during the intraoperative period ( - Fig. $\mathbf{3}$ ).

\section{Discussion}

This study demonstrated a close relationship between CVP and PVP, suggesting that PVP is a noninvasive, cost-effective, and simple procedure that can be used as a substitute for CVP monitoring. The results also revealed that PVP has a strong correlation with CVP, which is clinically acceptable. Our results are in line with the previous studies on this aspect of two pressure measurements. ${ }^{8-14}$

Since, peripheral veins have valves that may interrupt the continuous column of blood, are thin walled, and may be thus more easily subjected to compression and occlusion, use of PVP instead of CVP was not widely accepted in the past. ${ }^{15}$ To avoid any compression of the peripheral vein, we used peripheral access in right cephalic vein and the blood pressure cuff was attached on the other arm. Cephalic vein in antecubital fossa is relatively less liable to compression and occlusion than the more distal veins. Despite these fundamental drawbacks of peripheral veins, we observed a strong correlation between CVP and PVP. Studies have shown that 


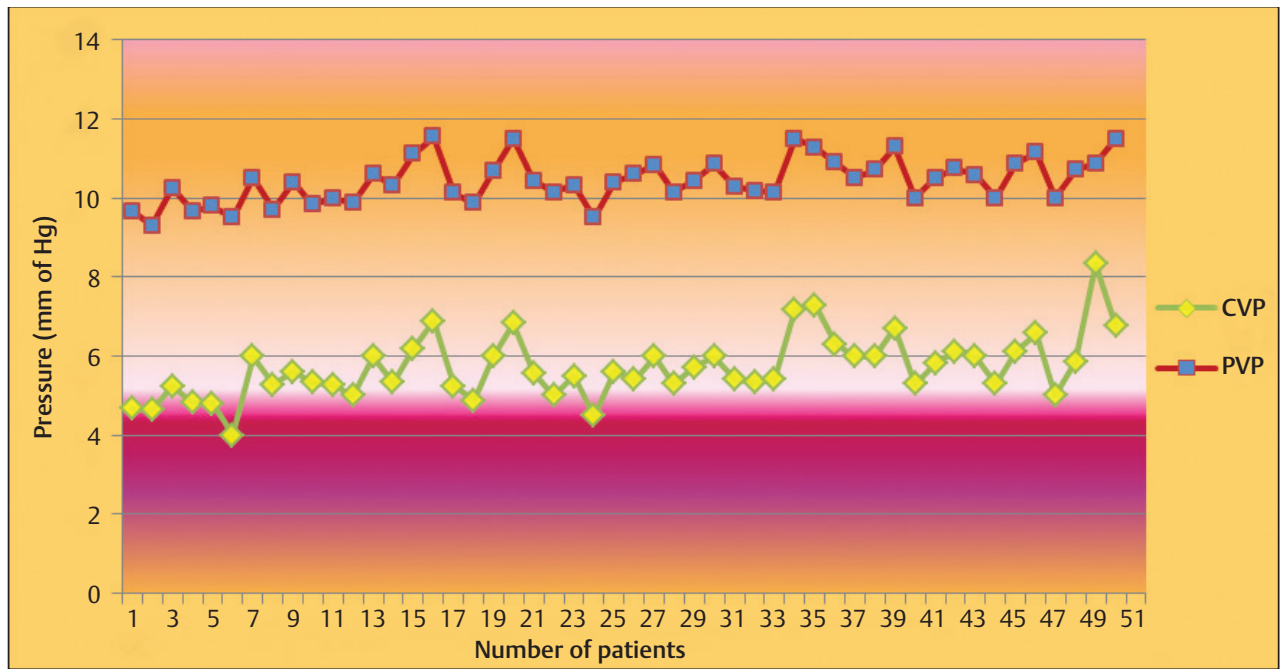

Fig. 1 Paired measurement of mean CVP and mean PVP. CPV, central venous pressure; PVP, peripheral venous pressure.

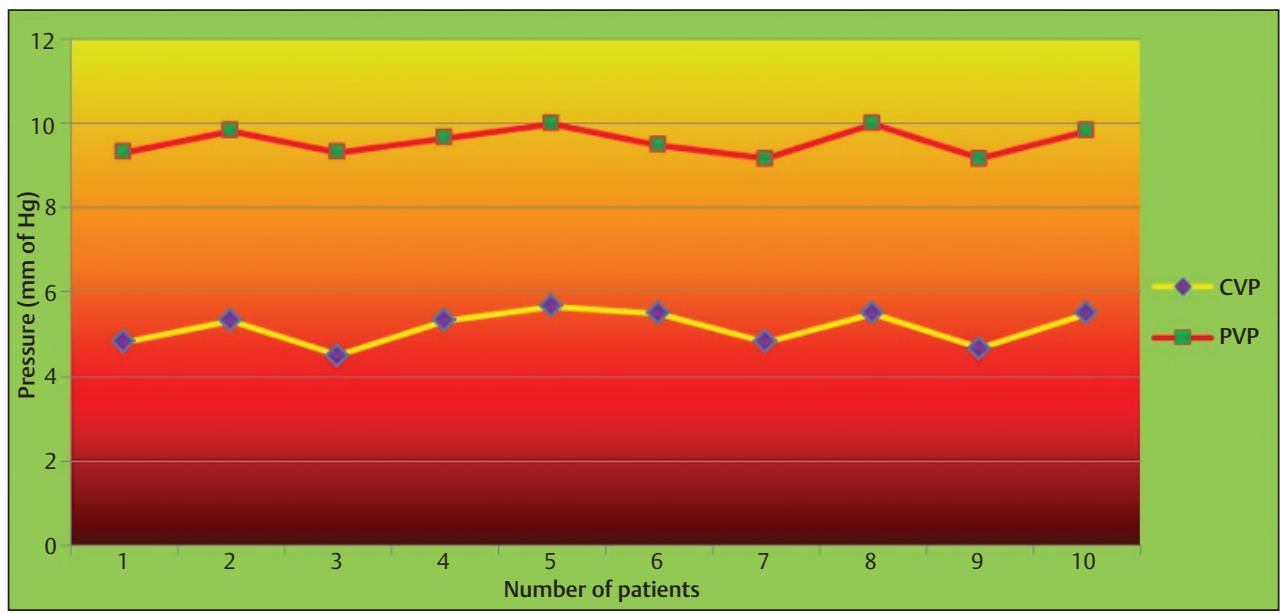

Fig. 2 Paired reading of mean CVP and mean PVP during hemodynamic instability. CPV, central venous pressure; PVP, peripheral venous pressure.

Table 4 Paired simultaneous reading of CVP and PVP in $\mathrm{mm}$ of $\mathrm{Hg}$ during hemodynamic instability

\begin{tabular}{|l|l|l|}
\hline Serial no. & Mean CVP $(\mathbf{m m}$ of $\mathrm{Hg})$ & Mean PVP $(\mathbf{m m}$ of $\mathrm{Hg})$ \\
\hline 1 & 4.83 & 9.33 \\
\hline 2 & 5.33 & 9.83 \\
\hline 3 & 4.5 & 9.33 \\
\hline 4 & 5.33 & 9.67 \\
\hline 5 & 5.67 & 10 \\
\hline 6 & 5.5 & 9.5 \\
\hline 7 & 4.83 & 9.17 \\
\hline 8 & 5.5 & 10 \\
\hline 9 & 4.67 & 9.17 \\
\hline 10 & 5.5 & 9.83 \\
\hline
\end{tabular}

Abbreviations: CVP, central venous pressure; PVP, peripheral venous pressure.

there is no difference in correlation between PVP and CVP with use of different sizes of peripheral intravenous catheter and also different sites of catheter placement in the arm. ${ }^{15}$ However, there is no consensus on this. ${ }^{11,14}$ To eliminate all these confounding factors, we placed same size cannula (that is 18 gauge) in the cephalic vein in all the patients.
Furthermore, our study revealed that not only it is possible to estimate CVP from PVP, but also the difference between CVP and PVP measurements remains almost in a constant range over a period of time. Similar observations have been reported in adult as well as in pediatric patients undergoing general surgical procedures. ${ }^{16,17}$ Amoozgar et al ${ }^{18}$ 


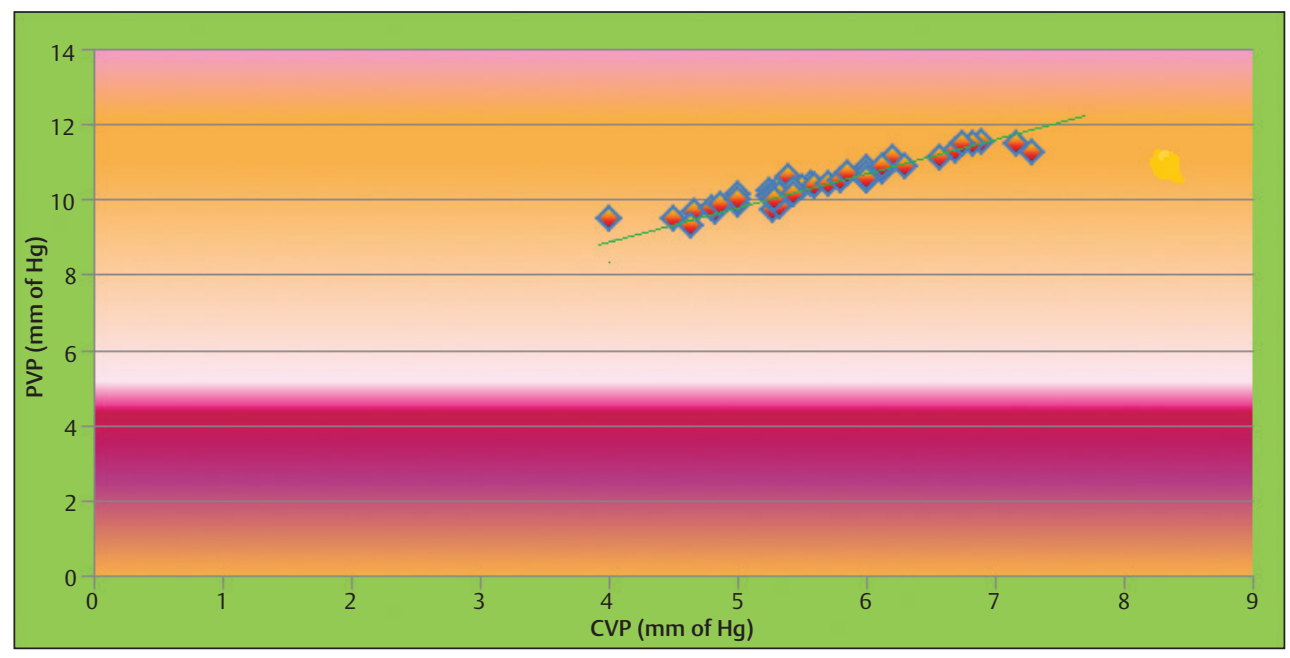

Fig. 3 Correlation between CVP and PVP during intraoperative period. CPV, central venous pressure; PVP, peripheral venous pressure.

concluded that even in infants and children with congenital heart disease (tetralogy of Fallot, pulmonary atresia, atrial septal defect, ventricular septal defect, patent ductus arteriosus, pulmonary stenosis, etc), PVP was a close reflection of CVP and its changes had similar trends over a period of time. Hence, all these studies emphasize that regardless of the disease condition (surgical or medical) there is a strong correlation between CVP and PVP. From all the above evidences, it can be safely concluded that irrespective of the diagnosis, age, sex, site, and size of insertion of the peripheral cannula, CVP and PVP correlate with each other. We observed that this relationship between CVP and PVP is not disturbed even during hemodynamic changes from blood loss; rather this correlation improves under such circumstances as was also observed by Munis et al. ${ }^{8}$ Given that the changes in CVP and PVP are strongly correlated, predictable and consistent over time, the trends in PVP may be useful for monitoring the intravascular volume status and fluid management.

Another benefit of monitoring PVP is that it maintains correlation with CVP even when patient is not in supine position. ${ }^{8}$ Therefore, in nonsupine positions where CVP may not give reliable information of volume status of a patient, monitoring PVP may be helpful, even during hemodynamic instability resulting from blood loss. ${ }^{9}$ We restricted our study to patients operated in supine position and standardized the sites of peripheral and central venous cannulation to minimize the error. Mean difference between CVP and PVP was $4.72 \pm 0.4(r=0.89,95 \% \mathrm{CI}:-4.61--4.83$, bias $=4.72$ and limit of agreement was 4.0 to $5.5 \mathrm{~mm}$ of $\mathrm{Hg}$ ), which was comparable with data described in other studies. However, this correlation is not strong in all types of surgical procedures, which was evident in orthotoptic liver transplantation. ${ }^{12}$ It could be because there is no single parameter that can guide fluid therapy in all circumstances.

There are some limitations to our study in that we confined our study to one site and one size of peripheral catheters in supine position only. We also did not compare the pressure gradient between CVP and PVP in patients with cardiac disease.
In conclusion, CVP and PVP measurements have strong correlation with each other and, PVP monitoring may be used as a noninvasive and cost-effective alternative to CVP for assessing volume status of neurosurgical patents operated in supine position, even during periods of significant blood loss.

\section{Funding \\ None.}

\section{Presentation at a Meeting}

The study was presented at the 28th Bihar-Jharkhand State Annual Conference of the Indian Society of Anaesthesiologists (ISA-BJSAC 2015) held from November 28 to 29 , 2015, Bhagalpur.

\section{Acknowledgments}

It is a privilege indeed to express my greatest regard and sincere gratitude to Dr. (Prof.) Radhika Dhanpal (HOD, VIMS \& RC, Bengaluru) and Dr. Rajesh Verma for their helpful attitude, readily available suggestions, profound interest and invaluable clinical tips, and encouragement at every step. I would also like to thank our operation theater staff for their help and assistance without which it would not have been possible to complete this study.

\section{Conflict of Interest}

None.

\section{References}

1 Magder S. How to use central venous pressure measurements. Curr Opin Crit Care 2005;11(3):264-270

2 Magder S. Central venous pressure monitoring. Curr Opin Crit Care 2006;12(3):219-227

3 Singh S, Kuschner WG, Lighthall G. Perioperative intravascular fluid assessment and monitoring: a narrative review of established and emerging techniques. Anesthesiol Res Pract 2011;2011:231493 doi:10.1155/2011/231493

4 McGee DC, Gould MK. Preventing complications of central venous catheterization. N Engl J Med 2003;348(12):1123-1133

5 Gelman S. Venous function and central venous pressure: a physiologic story. Anesthesiology 2008;108(4):735-748

6 Helmy A, Vizcaychipi M, Gupta AK. Traumatic brain injury: intensive care management. Br J Anaesth 2007;99(1):32-42 
7 Haddad SH, Arabi YM. Critical care management of severe traumatic brain injury in adults. Scand J Trauma Resusc Emerg Med 2012;20:12

8 Munis JR, Bhatia S, Lozada LJ. Peripheral venous pressure as a hemodynamic variable in neurosurgical patients. Anesth Analg 2001;92(1):172-179

9 Devaragudi TS, Rao GSU, Thennarasu K. Agreement of central venous pressure with peripheral venous pressure in neurosurgical patients. J Anaesthesiol Clin Pharmacol 2006;22:357-361

10 Anter AM, Bondok RS. Peripheral venous pressure is an alternative to central venous pressure in paediatric surgery patients. Acta Anaesthesiol Scand 2004;48(9):1101-1104

11 Desjardins R, Denault AY, Bélisle S, et al. Can peripheral venous pressure be interchangeable with central venous pressure in patients undergoing cardiac surgery? Intensive Care Med 2004;30(4):627-632

12 Hoftman N, Braunfeld M, Hoftman G, Mahajan A. Peripheral venous pressure as a predictor of central venous pressure during orthotopic liver transplantation. J ClinAnesth 2006;18(4):251-255
13 Hadimioglu N, Ertug Z, Yegin A, Sanli S, Gurkan A, Demirbas A. Correlation of peripheral venous pressure and central venous pressure in kidney recipients. Transplant Proc 2006;38(2):440-442

14 Tugrul M, Camci E, Pembeci K, Al-Darsani A, Telci L. Relationship between peripheral and central venous pressures in different patient positions, catheter sizes, and insertion sites. J Cardiothorac Vasc Anesth 2004;18(4):446-450

15 Charalambous C, Barker TA, Zipitis CS, et al. Comparison of peripheral and central venous pressures in critically Ill patients. Anaesth Intensive Care 2003;31(1):34-39

16 Amar D, Melendez JA, Zhang H, Dobres C, Leung DH, Padilla RE. Correlation of peripheral venous pressure and central venous pressure in surgical patients. J Cardiothorac Vasc Anesth 2001;15(1):40-43

17 Tobias JD, Johnson JO. Measurement of central venous pressure from a peripheral vein in infants and children. Pediatr Emerg Care 2003;19(6):428-430

18 Amoozgar H, Behniafard N, Borzoee M, Ajami GH. Correlation between peripheral and central venous pressures in children with congenital heart disease. Pediatr Cardiol 2008;29(2):281-284 